Global Journal of Pure and Applied Mathematics.

ISSN 0973-1768 Volume 13, Number 8 (2017), pp. 3951-3960

(C) Research India Publications

https://dx.doi.org/10.37622/GJPAM/13.8.2017.3951-3960

\title{
Two Warehouse Inventory Model with Different Deterioration Rates under Time Dependent Demand and Shortages
}

\author{
S.R. Sheikh \\ Department of Statistics \\ Prof.V.B. Shah Institute of Management \\ Amroli, Surat, India \\ Raman Patel \\ Department of Statistics \\ Veer Narmad South Gujarat University \\ Surat, INDIA
}

\begin{abstract}
An inventory model for two warehouses with different deterioration rates is developed. Demand is considered as linear function of time. Holding cost is time dependent. Shortages are allowed and completely backlogged. To support the model numerical case is given. Affectability investigation is likewise done for parameters.
\end{abstract}

Keywords: Two warehouse, deterioration, Linear demand, Time dependent holding cost, Shortages.

\section{INTRODUCTION:}

An item may be stocked in an inventory system at only a single physical location, or it may be stocked at many locations. In real life situation many time retailers decides to buy goods exceeding their Own Warehouse (OW) capacity to take advantage of price discounts. Therefore an additional stock is arranged and managed in Rented Warehouse (RW) which has better storage facilities with higher inventory carrying 
cost and low rate of deterioration. Bhunia and Maiti [1] proposed two warehouses inventory model for deteriorating items with linear demand and shortages. Goyal and Giri [2] presented recent trends in modeling of deteriorating inventory. Jaggi and Verma [4] suggested model with linear demand and inflationary conditions. Sana et al. [11] suggested model on pricing decision. Panda et al. [6] gave model with exponential demand and shortages. Skouri et al. [12] gave two warehouses model with ramp type demand. . Parekh and Patel [7] proposed two warehouse deteriorating item inventory model under inflation and permissible delay in payments. Patel and Parekh [8] proposed model for two warehouses under shortages, inflation and permissible delay in payments.

Palanivel and Uthaykumar [5] studied model for non-instantaneous deteriorating items with partial backlogging, inflation and finite time horizon. Jaggi et al. [3] gave replenishment policy for non-instantaneous deteriorating items under two storage facility and inflation. Raafat [9] and Ruxian et al.[10] gave review on deteriorating items inventory models.

Generally the products are such that there is no deterioration initially. After certain time deterioration starts and again after certain time the rate of deterioration increases with time. Here we have used such a concept and developed two warehouses deteriorating items inventory model.

In this paper we have developed a two warehouse inventory model with different deterioration rates. Demand function is linear, holding cost is time varying, Shortages are allowed and completely backlogged. Numerical case is given to represent the model. Affectability investigation is likewise done for parameters.

\section{ASSUMPTIONS AND NOTATIONS: NOTATIONS:}

The following notations are used for the development of the model:

$\mathrm{D}(\mathrm{t}) \quad$ : Demand rate is a linear function of time. $(\mathrm{a}+\mathrm{bt}, \mathrm{a}>0,0<\mathrm{b}<1)$

$\mathrm{HC}(\mathrm{OW})$ : Holding cost is linear function of time $\mathrm{t}\left(\mathrm{x}_{1}+\mathrm{y}_{1} \mathrm{t}, \mathrm{x}_{1}>0,0<\mathrm{y}_{1}<1\right)$ in OW.

$\mathrm{HC}(\mathrm{RW})$ : Holding cost is linear function of time $\mathrm{t}\left(\mathrm{x}_{2}+\mathrm{y}_{2} \mathrm{t}, \mathrm{x}_{2}>0,0<\mathrm{y}_{2}<1\right)$ in RW.

SC : shortage cost

A : Replenishment cost per order

c : Purchasing cost per unit

p : : Selling price per unit

$\mathrm{c}_{2} \quad$ : shortage cost per unit

$\mathrm{T} \quad$ : Length of inventory cycle 
$\mathrm{I}_{0}(\mathrm{t}) \quad$ : Inventory level in $\mathrm{OW}$ at time $\mathrm{t}$.

$\mathrm{I}_{\mathrm{r}}(\mathrm{t}) \quad$ : Inventory level in RW at time $\mathrm{t}$.

Q : Order quantity

$\mathrm{Q}_{1} \quad$ : Inventory level initially

$\mathrm{Q}_{2} \quad$ : shortage of inventory

$\mathrm{t}_{\mathrm{r}} \quad$ : time at which inventory level becomes zero in RW.

W : capacity of own warehouse

$\theta \quad$ : Deterioration rate during $\mu_{1} \leq \mathrm{t} \leq \mu_{2}, 0<\theta<1$

$\theta \mathrm{t} \quad:$ Deterioration rate during, $\mu_{2} \leq \mathrm{t} \leq \mathrm{t}_{0}, 0<\theta<1$

$\pi \quad$ : Total relevant profit per unit time.

\section{ASSUMPTIONS:}

The following assumptions are considered for the development of model.

- The demand of the product is declining as a linear function of time.

- Replenishment rate is infinite and instantaneous.

- Lead time is zero.

- Shortages are allowed and completely backlogged.

- OW has fixed capacity W units and RW has unlimited capacity.

- The goods of OW are consumed only after consuming the goods kept in RW.

- The unit inventory cost per unit in the RW is higher than those in the OW.

- Deteriorated units neither be repaired nor replaced during the cycle time.

\section{THE MATHEMATICAL MODEL AND ANALYSIS:}

At time $\mathrm{t}=0, \mathrm{Q}_{1}$ units enters into the system of which $\mathrm{W}$ are stored in $\mathrm{OW}$ and rest $\left(\mathrm{Q}_{1}-\mathrm{W}\right)$ are stored in $\mathrm{RW}$. During interval $\left(0, \mathrm{t}_{\mathrm{r}}\right)$ level of inventory in $\mathrm{RW}$ of depletes due to linear demand and reaches to 0 at time $t_{r}$ and inventory in OW remains $W$. During the interval $\left(t_{r}, \mu_{1}\right)$ inventory depletes in OW due to linear demand, during interval $\left(\mu_{1}, \mu_{2}\right)$ inventory depletes from OW due to deterioration at rate $\theta$ and linear demand. During interval $\left(\mu_{2}, t_{0}\right)$ inventory depletes due to joint effect of deterioration at rate $\theta \mathrm{t}$ and demand, By time $t_{0}$ both the warehouses are empty. Shortages occur during $\left(\mathrm{t}_{0}, \mathrm{~T}\right)$ of size $\mathrm{Q}_{2}$ units. The figure describes the behavior of inventory system. 
Let $\mathrm{I}(\mathrm{t})$ be the inventory at time $\mathrm{t}(0 \leq \mathrm{t} \leq \mathrm{T})$ as shown in figure.

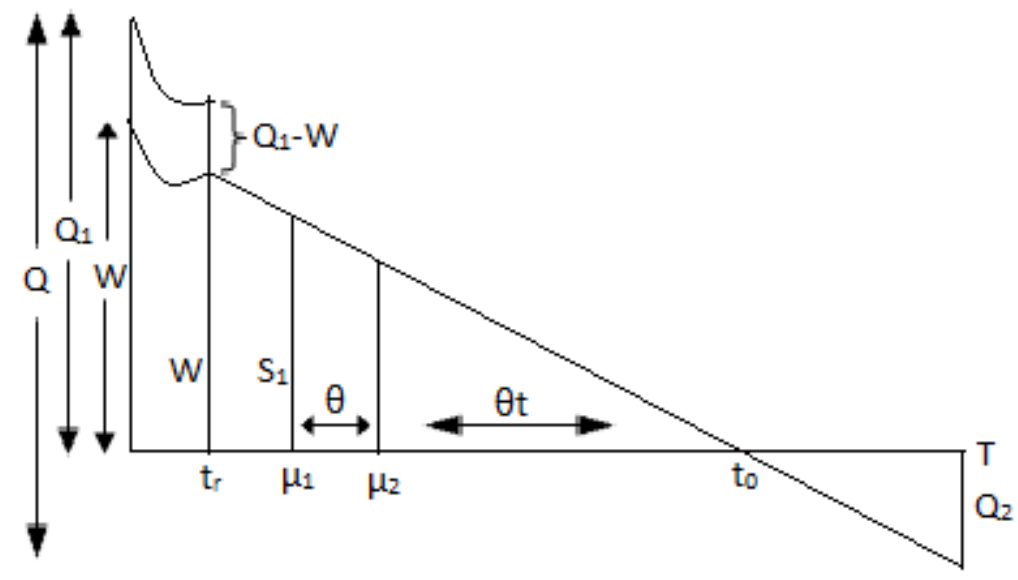

Figure 1

The differential equations which describes the instantaneous states of $I(t)$ over the period $(0, \mathrm{~T})$ is given by

$\frac{\mathrm{dI}_{\mathrm{r}}(\mathrm{t})}{\mathrm{dt}}=-(\mathrm{a}+\mathrm{bt})$

$$
0 \leq \mathrm{t} \leq \mathrm{t}_{\mathrm{r}}
$$

$\frac{\mathrm{dI}_{0}(\mathrm{t})}{\mathrm{dt}}=0$

$$
0 \leq \mathrm{t} \leq \mathrm{t}_{\mathrm{r}}
$$

$\frac{\mathrm{dI}_{0}(\mathrm{t})}{\mathrm{dt}}=-(\mathrm{a}+\mathrm{bt})$

$\mathrm{t}_{\mathrm{r}} \leq \mathrm{t} \leq \mu_{1}$

$\frac{\mathrm{dI}_{0}(\mathrm{t})}{\mathrm{dt}}+\theta \mathrm{I}_{0}(\mathrm{t})=-(\mathrm{a}+\mathrm{bt})$

$$
\mu_{1} \leq \mathrm{t} \leq \mu_{2}
$$

$\frac{d I_{0}(t)}{d t}+\theta I_{0}(t)=-(a+b t)$

$$
\mu_{2} \leq \mathrm{t} \leq \mathrm{t}_{0}
$$

$\frac{\mathrm{dI}_{\mathrm{s}}(\mathrm{t})}{\mathrm{dt}}=-(\mathrm{a}+\mathrm{bt})$

$$
\mathrm{t}_{0} \leq \mathrm{t} \leq \mathrm{T}
$$

With initial conditions

$\mathrm{I}_{0}(0)=\mathrm{W}, \mathrm{I}_{0}\left(\mu_{1}\right)=\mathrm{S}_{1}, \mathrm{I}_{0}\left(\mathrm{t}_{\mathrm{r}}\right)=\mathrm{W}, \mathrm{I}_{0}\left(\mathrm{t}_{0}\right)=0, \mathrm{I}_{\mathrm{r}}(0)=\mathrm{Q}_{1}-\mathrm{W}, \mathrm{I}_{\mathrm{r}}\left(\mathrm{t}_{\mathrm{r}}\right)=0, \mathrm{I}_{\mathrm{s}}(\mathrm{T})=-\mathrm{Q}_{2}$

Solutions of equations (1) to (6) are given by

$$
\begin{aligned}
& I_{r}(t)=Q_{1}-W-a t-\frac{1}{2} b t^{2} \\
& I_{0}(t)=W
\end{aligned}
$$




$$
\begin{aligned}
& I_{0}(t)=S_{1}+a\left(\mu_{1}-t\right)+\frac{1}{2} b\left(\mu_{1}^{2}-t^{2}\right) \\
& I_{0}(t)=\left\lfloor\begin{array}{l}
S_{1}\left(1+\theta\left(\mu_{1}-t\right)\right)+a\left(\mu_{1}-t\right)+\frac{1}{2} a \theta\left(\mu_{1}^{2}-t^{2}\right)+\frac{1}{2} b\left(\mu_{1}^{2}-t^{2}\right) \\
+\frac{1}{3} b \theta\left(\mu_{1}^{3}-t^{3}\right)-a \theta t\left(\mu_{1}-t\right)-\frac{1}{2} b \theta t\left(\mu_{1}^{2}-t^{2}\right)
\end{array}\right] \\
& I_{0}(t)=\left[\begin{array}{l}
a\left(t_{0}-t\right)+\frac{1}{6} a \theta\left(t_{0}{ }^{3}-t^{3}\right)+\frac{1}{2} b\left(t_{0}{ }^{2}-t^{2}\right) \\
+\frac{1}{8} b \theta\left(t_{0}{ }^{4}-t^{4}\right)-\frac{1}{2} a \theta t^{2}\left(t_{0}-t\right)-\frac{1}{4} b \theta t^{2}\left(t_{0}-t\right)
\end{array}\right\rfloor \\
& I_{0}(t)=a\left(t_{0}-t\right)+\frac{1}{2} b\left(t_{0}{ }^{2}-t^{2}\right)
\end{aligned}
$$

(by neglecting higher powers of $\theta$ )

Putting $\mu=t_{r}$ in equation (7) we get

$$
\mathrm{Q}_{1}=\mathrm{W}+\mathrm{at}_{\mathrm{r}}+\frac{1}{2} \mathrm{bt}_{\mathrm{r}}^{2}
$$

Putting $\mathrm{t}=\mathrm{T}$ in equation (12), we get

$$
\mathrm{Q}_{2}=\mathrm{a}\left(\mathrm{T}-\mathrm{t}_{0}\right)+\frac{1}{2} \mathrm{~b}\left(\mathrm{~T}^{2}-\mathrm{t}_{0}{ }^{2}\right)
$$

Putting $\mathrm{t}=\mathrm{t}_{\mathrm{r}}$ in (9) we have

$$
\mathrm{S}_{1}=\mathrm{W}-\mathrm{a}\left(\mu_{1}-\mathrm{t}_{\mathrm{r}}\right)-\frac{1}{2} \mathrm{~b}\left(\mu_{1}^{2}-\mathrm{t}_{\mathrm{r}}^{2}\right)
$$

From (10) and (11) putting $t=\mu_{2}$ we have

$$
\left.\begin{array}{l}
\mathrm{I}_{0}\left(\mu_{2}\right)=\left[\begin{array}{l}
\mathrm{S}_{1}\left(1+\theta\left(\mu_{1}-\mu_{2}\right)\right)+\mathrm{a}\left(\mu_{1}-\mu_{2}\right)+\frac{1}{2} \mathrm{a} \theta\left(\mu_{1}^{2}-\mu_{2}^{2}\right)+\frac{1}{2} \mathrm{~b}\left(\mu_{1}^{2}-\mu_{2}^{2}\right) \\
+\frac{1}{3} \mathrm{~b} \theta\left(\mu_{1}^{3}-\mu_{2}^{3}\right)-\mathrm{a} \theta \mu_{2}\left(\mu_{1}-\mu_{2}\right)-\frac{1}{2} \mathrm{~b} \theta \mu_{2}\left(\mu_{1}^{2}-\mu_{2}^{2}\right)
\end{array}\right] \\
\mathrm{I}_{0}\left(\mu_{2}\right)=\left\lfloor\begin{array}{l}
\mathrm{a}\left(\mathrm{t}_{0}-\mu_{2}\right)+\frac{1}{6} \mathrm{a} \theta\left(\mathrm{t}_{0}{ }^{3}-\mu_{2}{ }^{3}\right)+\frac{1}{2} \mathrm{~b}\left(\mathrm{t}_{0}{ }^{2}-\mu_{2}{ }^{2}\right) \\
+\frac{1}{8} \mathrm{~b} \theta\left(\mathrm{t}_{0}{ }^{4}-\mu_{2}{ }^{4}\right)-\frac{1}{2} \mathrm{a} \theta \mu_{2}{ }^{2}\left(\mathrm{t}_{0}-\mu_{2}\right)-\frac{1}{4} \mathrm{~b} \theta \mu_{2}{ }^{2}\left(\mathrm{t}_{0}-\mu_{2}\right)
\end{array}\right.
\end{array}\right]
$$


From (16) and (17) we have

$$
\mathrm{t}_{0}=\frac{\mathrm{S}_{1}\left(1+\theta\left(\mu_{1}-\mu_{2}\right)\right)+\mathrm{a} \mu_{1}}{\mathrm{a}}
$$

Based on the assumptions and descriptions of the model, the total annual relevant profit $(\pi)$, include the following elements:

(i) Ordering cost $(\mathrm{OC})=\mathrm{A}$

(ii) $\mathrm{HCRW}=\int_{0}^{\mathrm{t}_{\mathrm{r}}} \mathrm{I}_{\mathrm{r}}(\mathrm{t})\left(\mathrm{x}_{2}+\mathrm{y}_{2} \mathrm{t}\right) \mathrm{dt}$

$$
=\left\lfloor-\frac{1}{8} \mathrm{by}_{2} \mathrm{t}_{\mathrm{r}}{ }^{4}+\frac{1}{3}\left(-\mathrm{ay}_{2}-\frac{1}{2} \mathrm{bx}_{2}\right) \mathrm{t}_{\mathrm{r}}{ }^{3}+\frac{1}{2}\left(\left(\mathrm{at}_{\mathrm{r}}+\frac{1}{2} \mathrm{bt}_{\mathrm{r}}{ }^{2}\right) \mathrm{y}_{2}-\mathrm{ax}_{2}\right) \mathrm{t}_{\mathrm{r}}{ }^{2}+\left(a \mathrm{t}_{\mathrm{r}}+\frac{1}{2} \mathrm{bt}_{\mathrm{r}}{ }^{2}\right) \mathrm{x}_{2} \mathrm{t}_{\mathrm{r}}\right\rfloor
$$

(iii) $\mathrm{HCOW}=\int_{0}^{\mathrm{t}_{\mathrm{r}}} \mathrm{W}\left(\mathrm{x}_{1}+\mathrm{y}_{1} \mathrm{t}\right) \mathrm{dt}+\int_{\mathrm{t}_{\mathrm{r}}}^{\mu_{1}} \mathrm{I}_{0}(\mathrm{t})\left(\mathrm{x}_{1}+\mathrm{y}_{1} \mathrm{t}\right) \mathrm{dt}+\int_{\mu_{1}}^{\mu_{2}} \mathrm{I}_{0}(\mathrm{t})\left(\mathrm{x}_{1}+\mathrm{y}_{1} \mathrm{t}\right) \mathrm{dt}+\int_{\mu_{2}}^{\mathrm{t}_{0}} \mathrm{I}_{0}\left(\mathrm{x}_{1}+\mathrm{y}_{1} \mathrm{t}\right) \mathrm{dt}$

$=\left\lfloor\begin{array}{l}\frac{1}{2} \mathrm{Wy}_{1} \mathrm{t}_{\mathrm{r}}{ }^{2}+\mathrm{Wx}_{1} \mathrm{t}_{\mathrm{r}}-\frac{1}{8} \mathrm{by}_{1}\left(\mu_{1}^{4}-\mathrm{t}_{\mathrm{r}}^{4}\right)+\frac{1}{3}\left(-\mathrm{ay}_{1}-\frac{1}{2} \mathrm{bx}_{1}\right)\left(\mu_{1}^{3}-\mathrm{t}_{\mathrm{r}}^{3}\right)+ \\ \frac{1}{2}\left(\left(\mathrm{~S}_{1}+\mathrm{a} \mu_{1}+\frac{1}{2} \mathrm{~b}_{1}^{2}\right) \mathrm{y}_{1}-\mathrm{ax}_{1}\right)\left(\mu_{1}^{2}-\mathrm{t}_{\mathrm{r}}{ }^{2}\right)+\left(\mathrm{S}_{1}+\mathrm{a} \mu_{1}+\frac{1}{2} \mathrm{~b} \mu_{1}^{2}\right) \mathrm{x}_{1}\left(\mu_{1}-\mathrm{t}_{\mathrm{r}}\right)\end{array}\right\rfloor+$

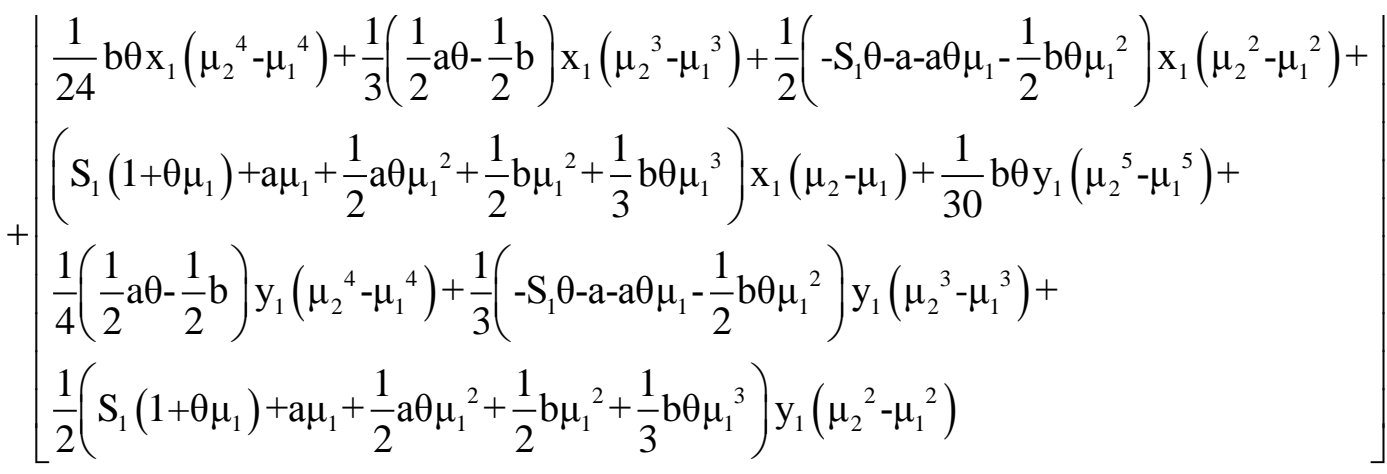

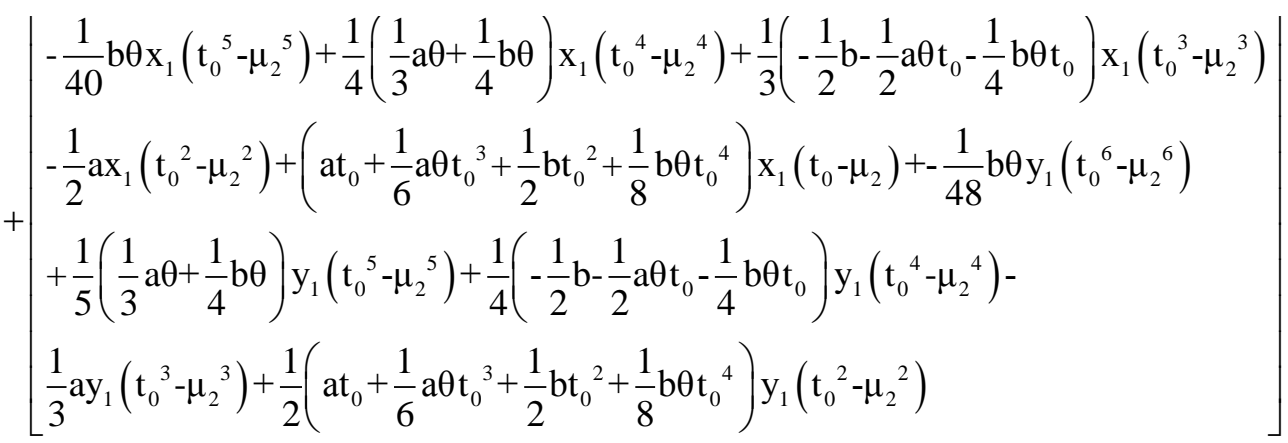


(iv) $\mathrm{DC}=\mathrm{c} \theta\left\lfloor\int_{\mu_{1}}^{\mu_{2}} \mathrm{I}_{0}(\mathrm{t}) \mathrm{dt}+\int_{\mu_{2}}^{\mathrm{t}_{0}} \mathrm{tI}_{0}(\mathrm{t}) \mathrm{dt}\right\rfloor$

$\mathrm{DC}=\left[\begin{array}{l}\mathrm{c} \theta\left(\begin{array}{l}-\frac{1}{48} \mathrm{~b} \theta\left(\mathrm{t}_{0}^{6}-\mu_{0}^{6}\right)+\frac{1}{5}\left(\frac{1}{3} \mathrm{a} \theta+\frac{1}{4} \mathrm{~b} \theta\right)\left(\mathrm{t}_{0}^{5}-\mu_{0}^{5}\right)+\frac{1}{4}\left(-\frac{1}{2} \mathrm{~b}-\frac{1}{2} \mathrm{a} \theta \mathrm{t}_{0}-\frac{1}{4} \mathrm{~b} \theta \mathrm{t}_{0}\right)\left(\mathrm{t}_{0}^{4}-\mu_{0}^{4}\right) \\ -\frac{1}{3} \mathrm{a}\left(\mathrm{t}_{0}{ }^{3}-\mu_{2}^{3}\right)+\frac{1}{2}\left(\mathrm{at}_{0}+\frac{1}{6} \mathrm{a} \theta \mathrm{t}_{0}^{3}+\frac{1}{2} \mathrm{bt}_{0}^{2}+\frac{1}{8} \mathrm{~b} \theta \mathrm{t}_{0}^{4}\right)\left(\mathrm{t}_{0}^{2}-\mu_{0}^{2}\right)\end{array}\right)\end{array}\right]$

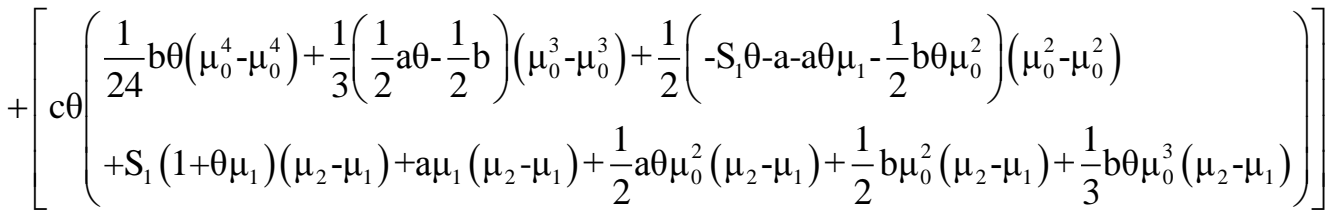

(v) $\mathrm{SC}=-\mathrm{c}_{2} \int_{\mathrm{t}_{0}}^{\mathrm{T}} \mathrm{I}_{\mathrm{s}}(\mathrm{t}) \mathrm{dt}$

$$
=\left\lfloor-c_{2}\left(-\frac{1}{6} b\left(T^{3}-t_{0}^{3}\right)-\frac{1}{2} a\left(T^{2}-t_{0}^{2}\right)+a_{0}\left(T-t_{0}\right)+\frac{1}{2} b_{0}^{2}\left(T-t_{0}\right)\right)\right\rfloor
$$

(vi) $\mathrm{SR}=\mathrm{p} \int_{0}^{\mathrm{T}}(\mathrm{a}+\mathrm{bt}) \mathrm{dt}=\mathrm{p}\left(\mathrm{aT}+\frac{1}{2} \mathrm{bT}^{2}\right)$

The total profit $(\pi)$ during a cycle, T consisted of the following:

$$
\pi=\frac{1}{\mathrm{~T}}[\mathrm{SR}-\mathrm{OC}-\mathrm{HC}(\mathrm{RW})-\mathrm{HC}(\mathrm{OW})-\mathrm{DC}-\mathrm{SC}]
$$

Substituting values from equations (19) to (24) in equation (25), we get total profit per unit. Putting $\mu_{1}=v_{1} T$ and $\mu_{2}=v_{2} T$ in equation (25), value of $S_{1}$ and $t_{0}$ from equation (15) and (18) in equation (25), we get profit in terms of $t_{r}$ and $\mathrm{T}$. Differentiating equation (25) with respect to $t_{r}$ and $T$ equate it to zero, we have

$$
\text { i.e. } \frac{\partial \pi\left(\mathrm{t}_{\mathrm{r}}, \mathrm{T}\right)}{\partial \mathrm{t}_{r}}=0, \frac{\partial \pi\left(\mathrm{t}_{\mathrm{r}}, \mathrm{T}\right)}{\partial \mathrm{T}}=0
$$

provided it satisfies the condition

$$
\left|\begin{array}{ll}
\frac{\partial^{2} \pi\left(\mathrm{t}_{\mathrm{r}}, \mathrm{T}\right)}{\partial \mathrm{t}_{\mathrm{r}}^{2}} & \frac{\partial^{2} \pi\left(\mathrm{t}_{\mathrm{r}}, \mathrm{T}\right)}{\partial \mathrm{t}_{\mathrm{r}} \partial \mathrm{T}} \\
\frac{\partial^{2} \pi\left(\mathrm{t}_{\mathrm{r}}, \mathrm{T}\right)}{\partial \mathrm{T} \partial \mathrm{t}_{\mathrm{r}}} & \frac{\partial^{2} \pi\left(\mathrm{t}_{\mathrm{r}}, \mathrm{T}\right)}{\partial \mathrm{T}^{2}}
\end{array}\right|>0
$$




\section{NUMERICAL EXAMPLE:}

Considering $A=R s .100, a=500, W=136, b=0.05, c=R s .25, c_{2}=8, p=$ Rs. 40, $\theta=0.05, \quad \mathrm{x}_{1}=$ Rs. $3, \mathrm{y}_{1}=0.05, \mathrm{x}_{2}=$ Rs. $6, \mathrm{y}_{2}=0.06, \mathrm{v}_{1}=0.30, \mathrm{v}_{2}=0.50$, in appropriate units. The optimal value of $\mathrm{t}_{\mathrm{r}}^{*}=0.0422, \mathrm{~T}^{*}=0.3751$, Profit $*=$ Rs. $19412.1471, \mathrm{Q}^{*}=$ 184.7190 .

The second order conditions given in equation (27) are also satisfied. The graphical representation of the concavity of the profit function is also given.

\begin{tabular}{|c|c|c|c|}
\hline & $\mathrm{t}_{\mathrm{r}}$ and Profit & $\mathrm{T}$ and Profit & $\mathrm{t}_{\mathrm{r}}, \mathrm{T}$ and profit \\
\hline & & & \\
\hline
\end{tabular}

\section{SENSITIVITY ANALYSIS:}

On the basis of the data given in example above we have studied the sensitivity analysis by changing the following parameters one at a time and keeping the rest fixed.

Table 1: Sensitivity Analysis

\begin{tabular}{|c|c|c|c|c|c|}
\hline Parameter & $\%$ & $\mathrm{t}_{\mathrm{r}}$ & $\mathrm{T}$ & Profit & $\mathrm{Q}$ \\
\hline \multirow{4}{*}{$\mathrm{a}$} & $+20 \%$ & 0.0504 & 0.3368 & 23455.7129 & 216.5420 \\
\cline { 2 - 6 } & $+10 \%$ & 0.0478 & 0.3545 & 21480.6888 & 200.7600 \\
\cline { 2 - 6 } & $-10 \%$ & 0.0395 & 0.3997 & 17533.5420 & 182.1710 \\
\cline { 2 - 6 } & $-20 \%$ & 0.0332 & 0.4296 & 15561.6658 & 175.5050 \\
\hline \multirow{4}{*}{$\theta$} & $+20 \%$ & 0.0422 & 0.3738 & 19503.5428 & 187.2760 \\
\cline { 2 - 6 } & $+10 \%$ & 0.0432 & 0.3744 & 19505.0571 & 187.5480 \\
\cline { 2 - 6 } & $-10 \%$ & 0.0453 & 0.3758 & 19508.1199 & 188.1900 \\
\cline { 2 - 6 } & $-20 \%$ & 0.0463 & 0.3765 & 19509.6798 & 188.5100 \\
\hline \multirow{4}{*}{$\mathrm{x}_{1}$} & $+20 \%$ & 0.0323 & 0.3692 & 19482.8180 & 184.8900 \\
\cline { 2 - 6 } & $+10 \%$ & 0.0383 & 0.3723 & 19494.5130 & 186.4550 \\
\cline { 2 - 6 } & $-10 \%$ & 0.0500 & 0.3777 & 19519.1002 & 189.1840 \\
\cline { 2 - 6 } & $-20 \%$ & 0.0556 & 0.3801 & 19531.9746 & 190.3980 \\
\hline
\end{tabular}




\begin{tabular}{|c|c|c|c|c|c|}
\hline \multirow{4}{*}{$\mathrm{x}_{2}$} & $+20 \%$ & 0.0376 & 0.3689 & 19505.2645 & 184.7530 \\
\cline { 2 - 6 } & $+10 \%$ & 0.0407 & 0.3718 & 19505.8861 & 186.2100 \\
\cline { 2 - 6 } & $-10 \%$ & 0.0485 & 0.3765 & 19507.4647 & 189.8800 \\
\cline { 2 - 6 } & $-20 \%$ & 0.0536 & 0.3840 & 19508.4884 & 192.3430 \\
\hline \multirow{4}{*}{$\mathrm{A}$} & $+20 \%$ & 0.0600 & 0.4037 & 19455.2585 & 202.2100 \\
\cline { 2 - 6 } & $+10 \%$ & 0.0523 & 0.3897 & 19480.4635 & 195.1900 \\
\cline { 2 - 6 } & $-10 \%$ & 0.0358 & 0.3600 & 19533.8137 & 180.2980 \\
\cline { 2 - 6 } & $-20 \%$ & 0.0271 & 0.3441 & 19562.2145 & 172.3270 \\
\hline \multirow{3}{*}{$c_{2}$} & $+20 \%$ & 0.0486 & 0.3618 & 19492.3950 & 181.2300 \\
\cline { 2 - 6 } & $+10 \%$ & 0.0466 & 0.3679 & 19499.0128 & 184.2750 \\
\cline { 2 - 6 } & $-10 \%$ & 0.0415 & 0.3837 & 19515.3731 & 192.1630 \\
\cline { 2 - 6 } & $-20 \%$ & 0.0384 & 0.3942 & 19525.6646 & 197.4050 \\
\hline
\end{tabular}

From the table we observe that as parameter a increases/ decreases average total profit increases/ decreases.

Also, we observe that with increase and decrease in the value of $\theta, x_{1}$, and $x_{2}$ there is corresponding decrease/ increase in total profit.

From the table we observe that as parameter A increases/ decreases average total profit also decreases/ increases.

\section{CONCLUSION:}

In this paper, we have developed a two warehouse inventory model for deteriorating items with different deterioration rates, linear demand and time varying holding cost with shortages. Sensitivity with respect to parameters has been carried out. The results show that with the increase/ decrease in the parameter values there is corresponding increase/ decrease in the value of profit.

\section{REFERENCES}

[1] Bhunia, A.K. and Maiti, M. (1998): A two-warehouse inventory model for deteriorating items with a linear trend in demand and shortages; J. of Oper. Res. Soc.; Vol. 49, pp. 287-292.

[2] Goyal, S.K. and Giri, B. (2001): Recent trends in modeling of deteriorating inventory; Euro. J. Oper. Res., Vol. 134, pp. 1-16.

[3] Jaggi, C.K., Tiwari, S. and Goel, S.K. (2016): Replenishment policy for noninstantaneous deteriorating items in a two storage facilities under inflationary conditions; Int. J. of industrial Engg. And Computations, Vol.7, pp. 489-506.

[4] Jaggi, C.K. and Verma, P. (2010): Two warehouse inventory model for deteriorating items with linear trend in demand and shortages under 
inflationary conditions; International J. of Procurement Management, Vol. 3, pp. 54-71.

[5] Palanivel, M. and Uthaykumar, R. (2015): Two warehouse inventory model for non instantaneous deteriorating items with partial backlogging and inflation over a finite time horizon; Opsearch, Vol. 52, pp. 1-25.

[6] Panda, G.C., Sahu, S. and Meher, M.K. (2012): A two warehouse inventory model for deteriorating items with exponential demand and shortages; American J. of Operational Research, Vol. 2(6), pp. 93-97.

[7] Parekh, R. U. and Patel, R. (2014): Deteriorating items inventory models for two warehouses with linear demand, time varying holding cost under inflation and permissible delay in payments; Int. J. of Math. And Statistics Invention, Vol. 2, pp. 39-48.

[8] Patel, R. and Parekh, R. (2014): Inventory model for variable Deteriorating items with two warehouses under shortages, time varying holding cost, inflation and permissible delay in payments; Int. Refereed Journal of Engineering and science (IRJES)., Vol. 3, No. 8, pp. 6-17.

[9] Raafat, F. (1991): Survey of literature on continuous deteriorating inventory model, J. of O.R. Soc., Vol. 42, pp. 27-37.

[10] Ruxian, L., Hongjie, L. and Mawhinney, J.R. (2010): A review on deteriorating inventory study; J. Service Sci. and management; Vol. 3, pp. 117-129

[11] Sana, S.S., Mondal, S.K., Sarkar, B.K. and Chaudhari, K. (2011): Two warehouse inventory model on pricing decision; International J. of Management Science and Engineering Management, Vol. 6(6), pp. 467-480.

[12] Skouri, K. and Konstantaras, I. (2013): Two- warehouse inventory model for deteriorating products with ramp type demand rate, J. of Ind. And Mgt. Opt., Vol, 9, No. 4, pp. 855-883. 